\title{
Experimental studies on fragmentation and exotic hadrons
}

\section{Kenkichi Miyabayashi*}

Department of Physics, Nara Women's University

E-mail: miyabayadcc.nara-wu.ac.jp

Formation of hadrons from quarks and gluons is the essential subject to understand the Quantum Chromo Dynamics (QCD) in its non-perturbative region. Measurements with the high statistics $e^{+} e^{-}$collision data have been providing important information by performing measurements of fragmentation and spectroscopy to reveal exotic hadrons' nature.

XXVI International Workshop on Deep-Inelastic Scattering and Related Subjects (DIS2018)

16-20 April 2018

Kobe, Japan

${ }^{*}$ Speaker. 


\section{Introduction}

Quantum Chromo Dynamics (QCD) is the basic theory to describe strong interaction. Its framework is based on the quantum field theory with $S U(3)$ gauge symmetry. High energy $e^{+} e^{-}$ and $e p$ colliders have been coming up with the measurements to confirm asymptotic freedom feature, i.e. running of the strong coupling $\alpha_{s}$, and to determine parton distribution function in the nucleon [ $[$ ]. These essential features of QCD in its perturbative region have been confirmed during the last decades, while still experimental inputs are desired for understanding about the low momentum transfer region where non-perturbative approaches must be employed as the $\alpha_{s}$ gets large and chiral symmetry is broken.

The $e^{+} e^{-}$colliding baem experiments can reveal the non-perturbative QCD characteristics in two different ways, measurements of fragmentation function and exotic hadron spectroscopy. For both of them, the Belle experiment at the KEKB asymmetric-energy $e^{+} e^{-}$collider brought a lot of results to enrich our knowledges. In this report, the Belle achievements in this area is reviewed and future prospects with higher statistics experiments is also mentioned.

\section{Fragmentation function measurements}

Fragmentation in the hadron jets produced by $e^{+} e^{-}$annihilation had been measured in various $e^{+} e^{-}$colliders such as DORIS, CESR, PEP, PETRA, TRISTAN and LEP in the last decades of 20th century. The data statistics recorded in these $e^{+} e^{-}$colliders are $\mathscr{O}\left(10^{4}\right) \sim \mathscr{O}\left(10^{6}\right)$ hadronic events. With such amount of data, initial quark's spin-averaged fragmentation function measurements were only possible. In the 21 st century, after the $B$-factory experiments, the BABAR experiment at SLAC and the Belle experiment at KEK were turned on, thanks to their outstanding high luminosity, $\mathscr{O}\left(10^{8}\right) \sim \mathscr{O}\left(10^{9}\right)$ hadronic events in $e^{+} e^{-}$annihilation at $\Upsilon$ region were recorded.

In order to probe the initial quark's spin dependence, the final state particles' azimuthal angle around the thrust axis in the event is chosen to see the transverse polarization with respect to the event plane spanned by the thrust and the incoming beam axis on the center-mass-frame. Dividing by the plane perpendicular to the thrust axis, two hemispheres are defined in a hadronic event. The azimuthal angle $\phi_{1}$ is defined as the angle of momentum vector $\vec{P}_{h 1}$ of the particle in the 1 st hemisphere with respect to the event plane. In the 2 nd hemisphere, $\phi_{2}$ is defined in the same manner. The modulation of particle production is measured as a function of the difference between $\phi_{1}$ and $\phi_{2}$ or $\phi_{1}+\phi_{2}$ [四]. Measurements for single particle as well as di-pion system [B] show that as the particle's momentum gets higher, the production rate modulation depending on azimuthal angle is larger. Invariant mass and fractional energy dependence of di-hadron production of $\pi \pi$ and $\pi K$ have also measured [ [ 9 ] .

High luminosity data collected at $B$-factory experiments also enable measurements for baryon production including various excited states in the large momentum range. Advantages with respect to the previous experiments is not only higher statistics but also high resolution charged particle tracking and calorimetry with particle identification capability. Measurements for hyperons and charmed baryons are carried out. Among charmed baryons, isosinglet states, $\Lambda_{c}, \Lambda_{c}(2595)$ and $\Lambda_{c}(2625)$, exhibit higher production rate than the ones isotriplet states, $\Sigma_{c}, \Sigma_{c}(2520)$ and $\Sigma_{c}(2800)$ [[]]. It indicates it is possible to probe the structure of hadrons by measuring their production rates. 


\section{Hadron spectroscopy and studies of exotic hadron candidates}

In QCD, there is no explicit forbidding rule to form unusual structure hadrons, i.e. not conventional $q \bar{q}$ meson states or $q q q$ baryon where $q$ denotes a constituent quark. The hadrons other than meson or baryon was discussed Gellman's classic paper. Such states are called exotic hadrons and several types have been discussed, tetraquark, pentaquark, meson-meson molecule, baryon-meson molecule and so on. However, we had lack of experimental evidence for the decades until the $B$-factory experiments were turned on.

\subsection{Studies of heavy quarkonium-like states}

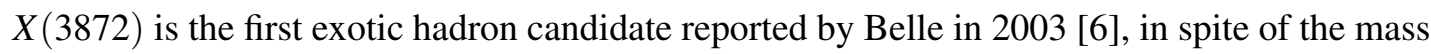
above $D \bar{D}$ threshold, it decays to $J / \psi \pi^{+} \pi^{-}$mode and is unusually narrow. Such characteristics are contradicting with the knowledge about the conventional charmonium. Its quantum numbers are determined to be $J^{P C}=1^{++}$by the radiative decay into $J / \psi \gamma$ and $\psi(2 S) \gamma[\square]$ as well as the decay product angular distribution in $J / \psi \pi^{+} \pi^{-}$mode [ []]. Since the mass is very close to the sum of $D^{0}$ and $D^{* 0}$ masses, the interpretation as a $D^{0} \bar{D}^{* 0}$ molecule is thought to be likely. Ii is known the ratio of branching fractions, $\mathscr{B}\left(X(3872) \rightarrow D^{0} \bar{D}^{* 0}\right) / \mathscr{B}\left(X(3872) \rightarrow J / \psi \pi^{+} \pi^{-}\right)$is about 10 [Q] , and there is direct production in high energy $p \bar{p}$ and $p p$ collisions at Tevatron and LHC [U]]. Currently the most plausible interpretation is that $X(3872)$ is an admixture of the meson-meson molecule and the charmonium component with the same quantum number [W], since it does not conflict all the available knowledges. Reaching at such an interpretation is regarded to be a substantial progress in the relevant research field.

The $Z_{b}(10610)^{ \pm}$and $Z_{b}(10650)^{ \pm}$are the charged bottomonium-like states which can only be explained by introducing four constituent quarks, $b \bar{b} u \bar{d}$. They are discovered in Belle data collected at $\Upsilon(10860)$ resonance [ㅁ] ]. For these states, $B^{*} \bar{B}^{(*)}$ decay mode is found to be dominant [[13] and their masses are so close to the $B^{*} \bar{B}^{(*)}$ thresholds, naturally those two charged $Z_{b}$ states are interpreted as the $B^{*} \bar{B}^{(*)}$ molecule [प14]. This picture also explains the fact the decay modes of $\Upsilon(1 S$ or $2 S$ or $3 S) \pi^{ \pm}$and $h_{b}(1 P$ or $2 P) \pi^{ \pm}$coexist, contrary to the usual bottomonium case in which one of them is strongly suppressed because heavy quark's spin flip is uneasy.

A counterpart in charmonium-like sector is the charged state $Z_{c}(3900)^{ \pm}$decaying to $J / \psi \pi^{ \pm}$ in the $Y(4260) \rightarrow J / \psi \pi^{+} \pi^{-}$process produced in the $e^{+} e^{-}$annihilation with initial state radiation (ISR) [प5]. HAL QCD group performed a simulation study and concluded that the $Z_{c}(3900)^{ \pm}$is likely to be a "Threshold cusp" [ए6].

Throughout these studies, near the threshold, meson-meson molecule is found to be playing very important role to form physically observed charmonium-like and bottomonium-like exotic candidate states. As seen in the cases of $Z_{b}(10610)^{ \pm}$and $Z_{b}(10650)^{ \pm}$at $\Upsilon(10860)$ ass well as $Z_{c}(3900)^{ \pm}$in $Y(4260)$ decay, $J^{P C}=1^{--}$heavy quakonium(-like) state above open charm or open bottom thresholds may contain the charged quakonium-like states which require four constituent quarks.

\subsection{Charm baryon excited states and related studies}

As an effective degree of freedom to form baryons, the diquark, which is strongly correlated two light quarks, is thought to play an appropriate role. However, in the nucleon case, the re- 
mained one constituent quark is also light, therefore strong correlation between particular two light quarks is not obvious. On the other hand, charm baryon case, because of the charm quark's much heavier mass than $u$ or $d$ quarks, its composition may be explained by one charm quark and a diquark. In order to see if such picture gives a proper description, it is necessary to identify various excited states. For the excited charm baryon states, the ratio of decay branching fractions between "ground state charm baryon + light meson(s)" mode(s) and "charm meson(s) + light baryon" mode(s) would give an important information to figure out possible interpretation. In Belle, $\Xi_{c}(3055)^{+}$and $\Xi_{c}(3080)^{+}$are found to decay both $\Lambda_{c}^{+} K^{-} \pi^{+}$and $\Lambda D^{+}$modes, while $\Xi_{c}(2980)^{+}$is confirmed only in $\Lambda_{c}^{+} K^{-} \pi^{+}$mode [ए]]. In general, most of excited state charm baryons have just started to be discivered, determination of their quantum numbers, $J^{P}$ needs higher statistics data.

For the first decade of 21 st century, high luminosity $e^{+} e^{-}$colliding beam experiments, BABAR and Belle, had lead relevant research field communities, and now LHCb experiment is also joining the game by utilizing high $b$ and $c$ hadron production in the forward direction in high energy $p p$ collision. The doubly charmed baryon, $\Xi_{c c}$ is seen in $\Lambda_{c}^{+} K^{-} \pi^{+} \pi^{+}$final state [एष]]. The $J / \psi p$ system in $\Lambda_{b} \rightarrow J / \psi p \pi^{-}$exhibits contributions due to the two pentaquark candidates [ए耳]. In these cases, background rejection is realized by exploiting significant vertex displacement from the primary $p p$ interaction thanks to larger boost and superb vertex detector. Since Belle II experiment with SuperKEKB $e^{+} e^{-}$collider at KEK will start data accumulation soon, for the measurements both $\mathrm{LHCb}$ and Belle II experiments can perform, friendly and exciting competition is anticipated. Each has also opportunities to perform its unique measurements.

\section{Summary}

To understand non-perturbative region phenomena of strong interaction, particularly hadron formation still inputs from experiments are desired. High luminosity $e^{+} e^{-}$colliding beam experiments give important information by performing fragmentation function measurements and heavy flavored hadron spectroscopy. Initial state quark's spin dependence of the fragmentation in $e^{+} e^{-}$ annihilation has been revealed by the analysis based on $\mathscr{O}\left(10^{8}\right)$ hadronic events. Spectroscopy of exotic hadrons for so-called $X Y Z$ states is providing interesting knowledge about the effective degrees of freedom to form hadrons. We expect friendly and exciting competition between Belle II and LHCb experiments ahead, hopefully we can reach deeper understanding about non-perturbative QCD.

\section{References}

[1] M. Tanabashi et al. Phys. Rev. D 98, 030001 (2018).

[2] R. Seidl et al. (Belle collaboration), Phys. Rev. Lett. 96, 232002 (2006); R. Seidl et al. (Belle collaboration), Phys. Rev. D 78, 032011 (2008).

[3] R. Seidl et al. (Belle collaboration), Phys. Rev. Lett. 96, 232002 (2011).

[4] R. Seidl et al. (Belle collaboration), Phys. Rev. D 96, 032005 (2017).

[5] M. Niiyama et al. (Belle collaboration), Phys. Rev. D 97, 072005 (2018).

[6] S. K. Choi, S. L. Olsen et al. (Belle Collaboration), Phys. Rev. Lett 91, 262001 (2003). 
[7] B. Aubert et al. (BABAR collaboration), Phys. Rev. Lett. 102, 132001 (2009); V. Bhardwaj et al. (Belle collaboration), Phys. Rev. Lett. 107, 091803 (2011); R. Aaji et al. (LHCb collaboration) Nucl. Phys. B 866665 (2014).

[8] S. K. Choi et al. (Belle Collaboration), Phys. Rev. D 84, 052004 (2011); A. Abulencia et al. (CDF II Collaboration), Phys. Rev. Lett 98, 132002 (2007); R. Aaji et al. (LHCb collaboration), Phys. Rev. Lett 110, 222001 (2013).

[9] T. Aushev et al. (Belle Collaboration), Phys. Rev. D 81, 031103 (2010); B. Aubert et al. (BaBar Collaboration), Phys. Rev. D 77, 011102 (2008).

[10] D. Acosta et al. (CDF II Collaboration), Phys. Rev. Lett 93, 072001 (2004); R. Aaij et al. (LHCb Collaboration), Eur. Phys. J. C 72, 1972 (2012).

[11] E. J. Eichiten et al. Phys. Rev. D 73, 014014 (2006); A. M. Badalin et al. Phys. Rev. D 85, 031103 (2012); S. Takeuchi, K. Shimizu and M.Takizawa PTEP2014, 123D01 (2014).

[12] A. Bonder et al. (Belle collaboration), Phys. Rev. Lett 108, 122001 (2012).

[13] A. Garmash et al. (Belle collaboration), Phys. Rev. Lett 116, 212001 (2016).

[14] A. Bonder et al. Phys. Rev. D 84, 054010 (2011).

[15] Z. Q. Liu et al. (Belle collaboration), Phys. Rev. Lett 110, 252002 (2013).

[16] Y. Ikeda et al. (HALQCD collaboration), Phys. Rev. Lett 117, 242001 (2016).

[17] Y. Kato et al. (Belle collaboration), Phys. Rev. D 89, 052003 (2014); Y. Kato et al. (Belle collaboration), Phys. Rev. D 94, 032002 (2016).

[18] R. Aaji et al. (LHCb collaboration), Phys. Rev. Lett 119, 112001 (2017).

[19] R. Aaji et al. (LHCb collaboration), Phys. Rev. Lett 117, 082003 (2016). 